\title{
组氨酸功能化碳点/石墨烯气凝胶的制备及超级电容器性能
}

\author{
丁卓峰 ${ }^{1}$, 杨永强 ${ }^{2}$, 李在均 ${ }^{1}$
}

(1. 江南大学 化学与材料工程学院, 无锡 214122; 2. 江苏省特种设备安全监督检验研究院 无锡分院, 无锡 214071)

摘 要: 通过一步水热法制备组氨酸功能化碳点/石墨烯气凝胶(His-CDs/GA)。该材料具有独特的三维多孔结构、丰 富的含氮和含氧官能团, 有利于电解液离子的快速扩散和提供更多的活性位点。当 GO 与 His-CDs 的质量比为 $2: 1$ 时, His-CDs/GA-2 在 $1 \mathrm{~A} \cdot \mathrm{g}^{-1}$ 电流密度下比电容达到 $304 \mathrm{~F} \cdot \mathrm{g}^{-1}$, 比 $\mathrm{GA}\left(172 \mathrm{~F} \cdot \mathrm{g}^{-1}\right)$ 提高了 $76.7 \%$; 当电流密度从 $1 \mathrm{~A} \cdot \mathrm{g}^{-1}$ 增加到 $50 \mathrm{~A} \cdot \mathrm{g}^{-1}$, 其比电容保持率为 $71.4 \%$; 在电流密度 $10 \mathrm{~A} \cdot \mathrm{g}^{-1}$ 下, 循环充放电 30000 次后, 比电容仍保留 $93.5 \%$ 。 由 His-CDs/GA 组装的对称超级电容器展现出高能量密度(在功率密度为 $250 \mathrm{~W} / \mathrm{kg}$ 时, 能量密度达到 $10.14 \mathrm{Wh} / \mathrm{kg}$ ) 和良好的循环性能(在 $5 \mathrm{~A} \cdot \mathrm{g}^{-1}$ 下循环充放电 20000 次后, 比电容保持率为 $88.4 \%$ )。结果表明, His-CDs/GA 是一种应 用前景广阔的超级电容器电极材料。

关 键 词: 石墨烯气凝胶; 组氨酸功能化碳点; 电容性能; 超级电容器

中图分类号: 0646 文献标识码: A

\section{Synthesis and Supercapacitor Performance of Histidine-functionalized Carbon Dots/Graphene Aerogel}

\author{
DING Zhuofeng ${ }^{1}$, YANG Yongqiang ${ }^{2}$, LI Zaijun ${ }^{1}$
}

(1. School of Chemical and Material Engineering, Jiangnan University, Wuxi 214122, China; 2. Jiangsu Province Special Equipment Safety Supervision and Inspection Institute Branch, Wuxi 214071, China)

\begin{abstract}
Histidine-functionalized carbon dot/graphene aerogel (His-CDs/GA) were synthesized via the one-step hydrothermal method. The as-prepared His-CDs/GA exhibits unique three-dimensional porous structure, rich nitrogen and oxygen-containing functional groups, which facilitate the rapid diffusion of electrolyte ions and provide more active sites. When the mass ratio of GO and His-CDs is $2: 1$, the specific capacitance of His-CDs/GA-2 reaches $304 \mathrm{~F} \cdot \mathrm{g}^{-1}$ at a current density of $1 \mathrm{~A} \cdot \mathrm{g}^{-1}$, which increases $76.7 \%$ compared with that of GA $\left(172 \mathrm{~F} \cdot \mathrm{g}^{-1}\right)$. With the current density increasing from $1 \mathrm{~A} \cdot \mathrm{g}^{-1}$ to $50 \mathrm{~A} \cdot \mathrm{g}^{-1}$, the specific capacitance retention of His-CDs/GA-2 achieves 71.4\%. The specific capacitance of His-CDs/GA-2 still remains $93.5 \%$ over 30000 cycles at $10 \mathrm{~A} \cdot \mathrm{g}^{-1}$. In addition, a symmetrical supercapacitor assembled by His-CDs/GA possesses a high energy density of $10.14 \mathrm{Wh} / \mathrm{kg}$ at a power density of $250 \mathrm{~W} / \mathrm{kg}$, and good cyclic performance with $88.4 \%$ capacitance retention at $5 \mathrm{~A} \cdot \mathrm{g}^{-1}$ over 20000 cycles. The results show that His-CDs/GA is a promising electrode material for supercapacitors.
\end{abstract}

Key words: graphene aerogel; histidine-functionalized carbon dot; capacitance performance; supercapacitor

收稿日期: 2019-12-11; 收到修改稿日期：2020-02-15

基金项目：国家科技重点研发计划(2018YFC1603001); 国家自然科学基金(21576115)

The National Key Research and Development Program of China (2018YFC1603001); National Natural Science Foundation of China (21576115)

作者简介: 丁卓峰(1996-), 男, 硕士研究生. E-mail: 1539342975@qq.com DING Zhuofeng(1996-), male, Master candidate. E-mail: 1539342975@qq.com

通讯作者: 李在均, 教授. E-mail: zaijunli@jiangnan.edu.cn LI Zaijun, professor. E-mail: zaijunli@jiangnan.edu.cn 
超级电容器具有充放电迅速、功率密度高、 循环寿命长等优点, 在储能领域中受到了广泛关 注 ${ }^{[1-2]}$ 。然而, 超级电容器的能量密度远低于二次电 池, 严重制约了其在电动汽车等需要较高能量密度 领域的应用 ${ }^{[3]}$ 。电极材料是提高超级电容器性能的 关键部分 ${ }^{[4]}$, 一般包括碳材料 ${ }^{[5]}$ 、过渡金属氧化物 ${ }^{[6]}$ 和导电聚合物 ${ }^{[7]}$ 。其中碳材料因其高比表面积、出 色的导电性和稳定性而得到了广泛应用 ${ }^{[8-10]}$ 。

在众多碳材料中，石墨烯具有大的理论比表面 积 $\left(2630 \mathrm{~m}^{2} \cdot \mathrm{g}^{-1}\right)$ 、优异的电导率以及低质量密度 ${ }^{[11]}$, 然而, 石墨烯片层之间强烈的 $\pi-\pi$ 相互作用使其易于 团聚, 导致实际比电容远低于理论值 $\left(550 \mathrm{~F} \cdot \mathrm{g}^{-1}\right)^{[12]}$ 。 许多研究致力于制备三维多孔石墨烯或将碳材料引 入到石墨烯中来解决该问题。比如, Sun 等 ${ }^{[13]}$ 制备的 氮掺杂石墨烯气凝胶, 在 $0.25 \mathrm{~A} \cdot \mathrm{g}^{-1}$ 电流密度下, 比 电容达到 $204 \mathrm{~F} \cdot \mathrm{g}^{-1}$ 。Zhao 等 ${ }^{[14]}$ 将介孔碳球引入到石 墨烯中, 有效抑制了石墨烯片的团聚, 在 $1 \mathrm{~A} \cdot \mathrm{g}^{-1}$ 下, 比电容达到 $242.3 \mathrm{~F} \cdot \mathrm{g}^{-1}$ 。然而, 石墨烯基电极材料 的电容性能仍难以满足实际应用的需求, 需要进一 步设计开发高性能的石墨烯基电极材料。

碳点 $(\mathrm{CDs})$ 作为一类新型的零维碳材料(尺寸一 般小于 $10 \mathrm{~nm}$ ), 不仅来源广泛、毒性低、环境友好 和化学性质稳定，而且具有独特的量子限域效应、 边缘效应和荧光效应等特性, 被广泛应用在生物成 像、传感器、光电催化等领域 ${ }^{[15-17]}$ 。小尺寸的 CDs 具有丰富的边缘位点和官能团, 且易功能化、引入 特殊的活性基团, 能够作为纳米填充剂提升复合材 料的电化学性能 ${ }^{[18-20]}$ 。比如, Wei 等 ${ }^{[19]}$ 采用浸渍-煅 烧法合成氮氧磷共掺杂碳点/多孔碳复合材料。本课 题组 ${ }^{[20]}$ 制备了色氨酸功能化石墨烯量子点 $/ \mathrm{NiCo}_{2} \mathrm{~S}_{4}$, 色氨酸功能化石墨烯量子点的引入显著改善了复合 材料的电子/离子传导率。

本研究以柠檬酸和组氨酸为原料, 采用热裂解 法制备出组氨酸功能化碳点(His-CDs), 再通过一步 水热法制备出组氨酸功能化碳点/石墨烯气凝胶 (His-CDs/GA), 并系统研究 His-CDs 添加量对复合 材料电容性能的影响, 以期得到高比容量、倍率性 能和循环性能的电极材料。

\section{1 实验方法}

\subsection{His-CDs 的制备}

将 $2.10 \mathrm{~g}$ 柠檬酸和 $1.55 \mathrm{~g}$ 组氨酸通过超声溶解 在 $20 \mathrm{~mL}$ 的去离子水中, 于 $80{ }^{\circ} \mathrm{C}$ 下蒸干水分。将 得到的粘稠浆液转移到烘箱, $180{ }^{\circ} \mathrm{C}$ 下反应 $3 \mathrm{~h}$ 。将 获得的粉末分散在去离子水中, 用 $1 \mathrm{~mol} \cdot \mathrm{L}^{-1} \mathrm{NaOH}$
溶液调节 $\mathrm{pH}$ 至 7 , 透析 $24 \mathrm{~h}$ 除去未反应完全的原 料。最后冷冻干燥 $48 \mathrm{~h}$ 得到 His-CDs 固体粉末。

\section{$1.2 \mathrm{His}-\mathrm{CDs} / \mathrm{GA}$ 的制备}

采用改良的 Hummers 法制备氧化石墨烯 $(\mathrm{GO})^{[2]}$ 。 称取 $40 \mathrm{mg} \mathrm{GO}$ 分散在 $40 \mathrm{~mL}$ 去离子水中, 超声 $3 \mathrm{~h}$ 。 将一定质量的 His-CDs 分散在 GO 分散液中, 快速 搅拌 $10 \mathrm{~min}$, 然后超声 $30 \mathrm{~min}$ 。将获得的 His-CDs/ $\mathrm{GA}$ 分散液转移到高压反应釜中, 于 $180{ }^{\circ} \mathrm{C}$ 反应 $12 \mathrm{~h}$ 。 冷却至室温后洗涤。最后通过冷冻干燥获得 His$\mathrm{CDs} / \mathrm{GA}$ 。为研究 His-CDs 添加量对复合材料电化学 性能的影响, 分别制备了 GO:His-CDs 质量比为 $4: 1 、 2: 1$ 和 $1: 1$ 的样品, 依次记为 His-CDs/GA-1、 His-CDs/GA-2 和 His-CDs/GA-3。并在相同的条件 下不添加 His-CDs, 制备 GA 作为对比。

\section{3 样品表征与性能测试}

采用 S4800 型场发射扫描电子显微镜(日本日 立株式会社)观察形貌, 测试电压为 $3 \mathrm{kV}$; 采用 JEM2100 型透射电子显微镜(日本 JEOL 公司)观察微观 结构, 测试电压为 $200 \mathrm{kV}$; 采用 D8 Advance 型 X 射 线衍射仪(德国 Bruker AXS 公司)分析结构, $\mathrm{Cu}$ 靶 $\mathrm{K} \alpha$ 射线, 扫描范围为 $10^{\circ} \sim 70^{\circ}$, 扫描速率为 $2\left(^{\circ}\right) / \mathrm{min}$; $\mathrm{N}_{2}$ 吸附/脱附测试采用 Tristar II 3020 型比表面仪(美 国 Micromeritics 公司); XPS 测试采用 Axis supra 型 $X$ 射线光电子能谱仪(英国 Kratos 公司); 拉曼光谱测 试采用 InVia 型显微共焦激光拉曼光谱仪(法国 Jobin-Yvon 公司); 红外光谱测试采用 Nicolet FT-IR 6700 型红外光谱仪(美国赛默飞世尔科技公司); 循 环伏安 $(\mathrm{CV})$ 、恒电流充放电 $(\mathrm{GCD})$ 和电化学阻抗 (EIS)测试采用 CHI 660D 型电化学工作站(上海辰华 有限公司)(具体测试方法详见补充材料 S1)。

\section{2 结果与讨论}

\subsection{His-CDs/GA 的制备过程}

His-CDs/GA 的制备示意图如图 1 所示, 分别以 柠檬酸和组氨酸为原料, 采用热裂解法制备出 His-CDs, 尺寸为 $3 \sim 8 \mathrm{~nm}$, 其边缘具有丰富的含氧 官能团, 如羧基 $(-\mathrm{COOH})$, 以及含氮官能团, 如氨 基- $\left.\mathrm{NH}_{2}\right)$ 和咪唑基。His-CDs 边缘的氨基 $\left(-\mathrm{NH}_{2}\right)$ 和羧 基(- $\mathrm{COOH}$ )能够与 $\mathrm{GO}$ 边缘的羧基(- $\mathrm{COOH})$ 和羟基 $(-\mathrm{OH})$ 产生氢键作用 $(\mathrm{N}-\mathrm{H} \cdots \mathrm{O} 、 \mathrm{O}-\mathrm{H} \cdots \mathrm{O})$, 并在水 热过程中发生脱水缩合反应，从而负载在石墨烯片 上形成 His-CDs/GA 复合材料 ${ }^{[12]}$ 。此外, 水热反应 能够去除 GO 和 His-CDs 大部分的含氧基团, 提高 材料的导电性, 而 GO 和 His-CDs 剩余的含氧基团 有助于提高比电容 ${ }^{[21]}$ 。 


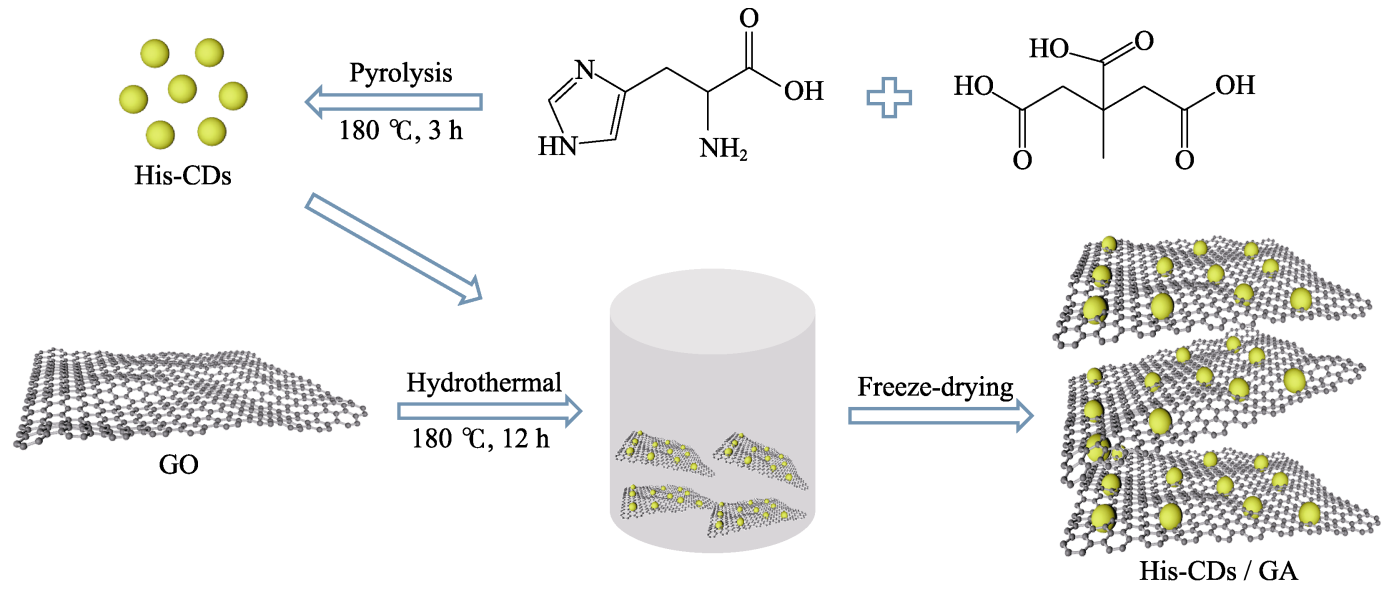

图 1 His-CDs/GA 的制备示意图

Fig. 1 Schematic illustration of His-CDs/GA preparation

\section{2 结构与形貌表征}

图 2 为 His-CDs 的 TEM 照片, His-CDs 颗粒分 布均匀, 无明显团聚。从图 2 的插图中观察到单个 His-CDs 的晶格条纹间距为 $0.21 \mathrm{~nm}$, 对应于石墨的 (100)晶面。图 S1 为 His-CDs 的尺寸分布图, 其尺寸 在 3 8 $\mathrm{nm}$ 之间。图 S2(a)为 His-CDs 的 XRD 图谱, 在 $2 \theta=25.1^{\circ}$ 处的衍射宽峰归因于高度缺陷或无序的 碳 ${ }^{[15]}$ 。图 S2(b)为 His-CDs 的红外光谱图, $3149 \mathrm{~cm}^{-1}$ 附近的吸收峰归属于 $\mathrm{NH}_{2}$ 的伸缩振动; $1696 \mathrm{~cm}^{-1}$ 处 的强峰属于 $\mathrm{C}=\mathrm{O}$ 键的伸缩振动; $1590 \mathrm{~cm}^{-1}$ 处的吸收 峰属于 $\mathrm{C}=\mathrm{N}$ 键的伸缩振动, 表明 His-CDs/GA 含有 咪唑基; $1386 \mathrm{~cm}^{-1}$ 处的峰属于 $\mathrm{C}-\mathrm{H}$ 键或 $-\mathrm{C}-\mathrm{N}$; $1160 \mathrm{~cm}^{-1}$ 处的吸收峰归属于 $\mathrm{C}-\mathrm{O}$ 键伸缩振动。以 上表征证实成功制备出 His-CDs。

图 3(a d) 分别为 GA、His-CDs/GA-1、His-CDs/ GA-2 和 His-CDs/GA-3 的 SEM 照片。它们均由褶 皱弯曲的石墨烯纳米片通过 $\pi-\pi$ 堆叠和氢键作用形 成了相互交联的三维网络结构 ${ }^{[2]}$ 。相比较于 GA, His-CDs/GA 片层的团聚明显减少, 说明将 His-CDs 负载在 GA 上能够有效抑制石墨烯片的团聚。GA 的 TEM 照片(图 3(e))进一步显示其纳米片相互堆积在

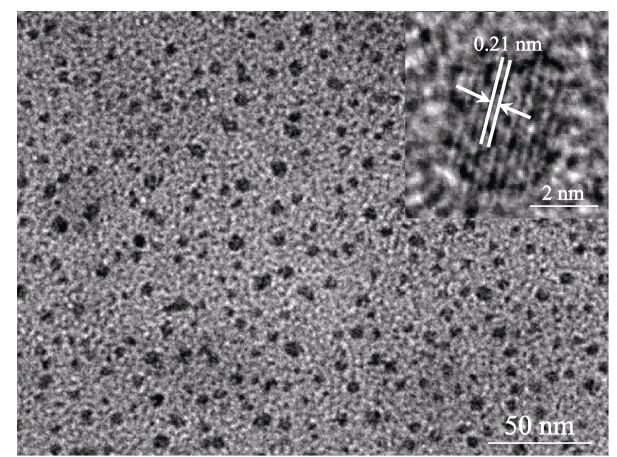

图 2 His-CDs 的 TEM 照片(插图: HRTEM 照片)

Fig. 2 TEM image of His-CDs (Inset: HRTEM image)
一起。图 3(f)为 His-CDs/GA-2 的 TEM 照片。His-CDs 均匀针定在超薄的石墨烯纳米片上, 说明石墨烯纳 米片同样能够阻碍 His-CDs 的团聚。

图 4 为样品的 XRD 图谱。图中 $\mathrm{GA}$ 位于 $2 \theta=25.3^{\circ}$ 处、His-CDs/GA- 1 位于 $2 \theta=24.7^{\circ}$ 处、His-CDs/GA-2 位于 $2 \theta=24.6^{\circ}$ 处和 His-CDs/GA-3 位于 $2 \theta=24.9^{\circ}$ 处出 现的衍射峰均对应于石墨烯的(002)晶面, 由布拉格 方程计算出其对应的层间距分别为 $0.352 、 0.360$ 、 0.362 和 $0.357 \mathrm{~nm}$ 。相对于 GA, His-CDs/GA-1、
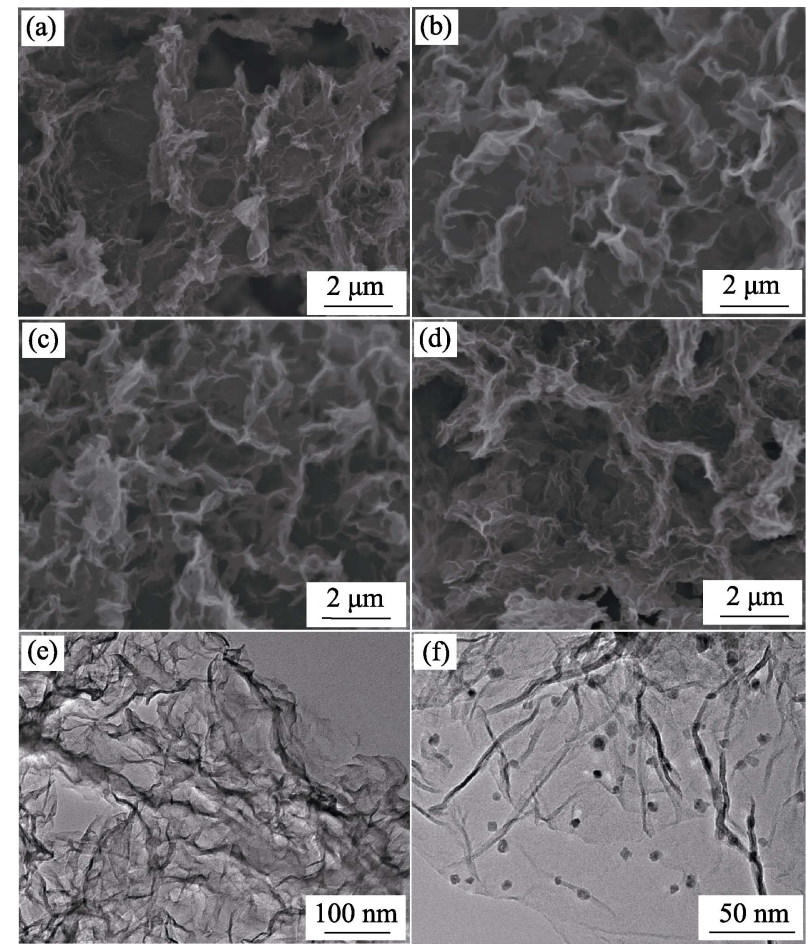

图 3 GA(a)、His-CDs/GA-1(b)、His-CDs/GA-2(c)和 His-CDs/ GA-3(d)的 SEM 照片; GA(e)和 His-CDs/GA-2(f)的 TEM 照片 Fig. 3 SEM images of GA(a), His-CDs/GA-1(b), His-CDs/ GA-2(c), and His-CDs/GA-3(d), and TEM images of GA(e) and His-CDs/GA-2(f) 


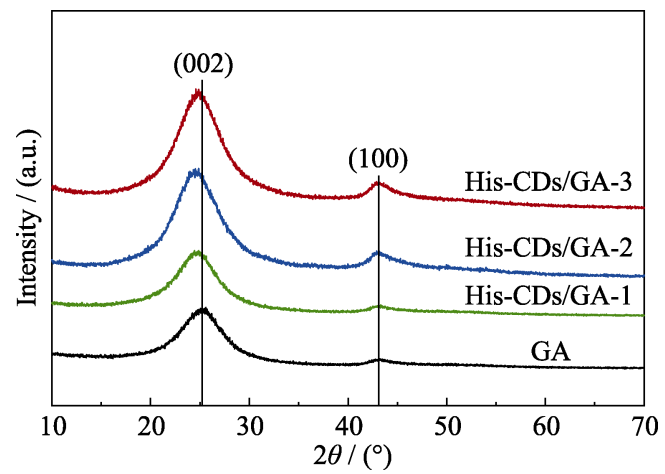

图 4 GA、His-CDs/GA-1、His-CDs/GA-2 和 His-CDs/GA-3 的 XRD 图谱

Fig. 4 XRD patterns of GA, His-CDs/GA-1, His-CDs/GA-2 and His-CDs/GA-3

His-CDs/GA-2 和 His-CDs/GA-3 的层间距都有不同 程度的提高, 说明 His-CD 的引入能够增加石墨烯 片的层间距，这有利于电解液离子的快速传输 ${ }^{[11]}$ 。 另外, 四个样品在 $2 \theta=43.2^{\circ}$ 处的弱衍射峰, 对应于石 墨烯的(100)晶面。样品的拉曼光谱图如图 S3 所示, $1350 \mathrm{~cm}^{-1}$ 处的 D 带来自于样品中无序和缺陷的 $\mathrm{sp}^{3}$ 杂化的碳, $1600 \mathrm{~cm}^{-1}$ 处的 $\mathrm{G}$ 带来自于样品中 $\mathrm{sp}^{2}$ 杂 化的石墨化碳 ${ }^{[10]}$ 。His-CDs/GA-1、His-CDs/GA-2 和 His-CDs/GA-3 的 D 带与 $\mathrm{G}$ 带的强度比 $\left(I_{\mathrm{D}} / I_{\mathrm{G}}\right)$ 分别为 1.02、1.04 和 1.03 , 均略高于 $\mathrm{GA}(0.99)$, 表明引入 His-CDs 能够增加复合材料的缺陷并加剧无序化程 度, 从而提供更多的电化学活性和缺陷位点 ${ }^{[22]}$ 。

GA、His-CDs/GA-1、His-CDs/GA-2 和 His-CDs/ GA-3 的氮气吸附/脱附等温线如图 S4(a)所示。4 种 样品在相对压力 $\left(p / p_{0}\right)$ 为 $0.4 \sim 1.0$ 之间均出现明显的 滞后环，具有典型的 IV 型特征，说明样品均存在介 孔结构 ${ }^{[8]}$ 。GA、His-CDs/GA-1、His-CDs/GA-2 和 His-CDs/GA-3 的比表面积分别为 142.4、247.5、 295.2 和 $199.8 \mathrm{~m}^{2} \cdot \mathrm{g}^{-1}$ ，孔体积分别为 $0.16 、 0.23 、 0.25$ 和 $0.21 \mathrm{~cm}^{3} \cdot \mathrm{g}^{-1}$ 。相比较于 $\mathrm{GA}$, 复合材料的比表面积 明显提高, 这是由于负载在石墨烯片上的 His-CDs 有效抑制了石墨烯片的团聚。图 S4(b)为四种样品的 孔径分布曲线。 4 种样品的介孔尺寸都主要分布在 15 20 和 30 40 $\mathrm{nm}$ 之间, 且 His-CDs/GA 的孔体积 高于 GA。这主要归因于以下两点: (1)His-CDs 被固 定在石墨烯片的表面及边缘，显著增加了石墨烯片 之间相互作用的位点, 促进凝胶形成过程中产生更 多的介孔，因此介孔的孔体积明显增大；(2)石墨烯 气凝胶孔径分布规律主要取决于石墨烯片的大小、 功能基团和凝胶化工艺, 碳点的作用十分有限。

图 5(a)为 GA 和 His-CDs/GA-2 的 XPS 全谱。 $\mathrm{GA}$ 在 284 和 $531 \mathrm{eV}$ 处出现两个不同的峰, 分别归属 于 $\mathrm{C} 1 \mathrm{~s}$ 和 $\mathrm{O} 1 \mathrm{~s} 。 \mathrm{His}-\mathrm{CDs} / \mathrm{GA}-2$ 于 284、399 和 $531 \mathrm{eV}$
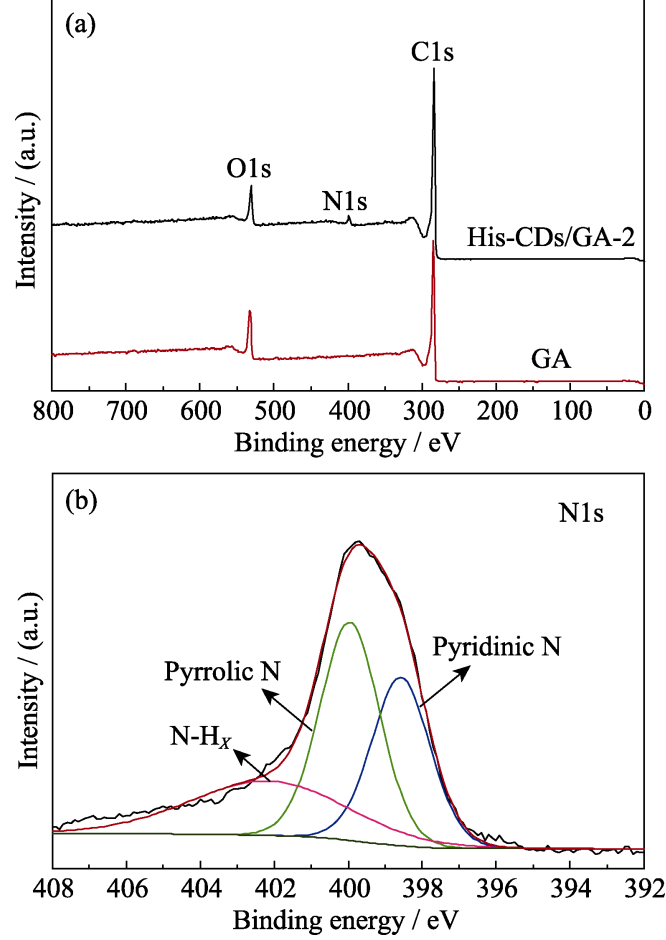

图 $5 \mathrm{GA}$ 和 His-CDs/GA-2 的 XPS 总谱(a)和 His-CDs/GA-2 的 N 1s 图谱(b)

Fig. 5 Total survey XPS spectra (a) of GA and His-CDs/GA-2 and $\mathrm{N}$ 1s spectrum (b) of His-CDs/GA-2

处的峰分别归属于 $\mathrm{C} 1 \mathrm{~s} 、 \mathrm{~N} 1 \mathrm{~s}$ 和 $\mathrm{O} 1 \mathrm{~s}$ ，其中氮元素的 含量为 $3.58 \mathrm{at} \%$ 。相比较于 GA, His-CDs/GA-2 出现 了 N1s 峰, 说明 His-CDs 和 GA 成功结合。图 5(b) 为 His-CDs/GA-2 的 N1s 谱图。在 N 1s 光谱中存在 3 个峰, 398.6、400.0 和 $402.2 \mathrm{eV}$ 处的峰分别对应于 吡啶氮、吡咯氮和 $\mathrm{N}-\mathrm{H}_{x}{ }^{[11]}$ 。吡啶氮和吡咯氮表示 $\mathrm{N}$ 原子取代了五元环中的 $\mathrm{C}$ 原子，能够给碳骨架造成 大量的缺陷。这有利于增强赝电容性能，从而提高 电极的整体比电容 ${ }^{[5]}$ 。此外, 吡啶氮具有更大的偶极 矩, 能够降低活性材料和电解质界面处的电荷转移电 阻 ${ }^{[23]}$ 。His-CDs/GA-2 的 $\mathrm{C} 1 \mathrm{~s}$ 和 O1s 谱图如图 S5(a b) 所示。 $\mathrm{C} 1 \mathrm{~s}$ 光谱中, 284.8、285.4、286.5 和 $287.8 \mathrm{eV}$ 处的峰分别对应于 $\mathrm{C}$ 不同的化学键 $\mathrm{C}=\mathrm{C} / \mathrm{C}-\mathrm{C} 、 \mathrm{C}-\mathrm{N}$ 、 $\mathrm{C}-\mathrm{O}$ 和 $\mathrm{C}=\mathrm{O}^{[5]}$ 。在 $\mathrm{O} 1 \mathrm{~s}$ 光谱中, 在 530.6、531.4 和 $533.4 \mathrm{eV}$ 处的峰分别归属于 $\mathrm{O}-\mathrm{H} 、 \mathrm{C}=\mathrm{O}$ 和 $\mathrm{C}-\mathrm{O}-\mathrm{C} /$ $\mathrm{C}-\mathrm{OH}^{[10]}$, 证实水热反应后样品还保留了部分含氧 基团。

\section{3 三电极电化学性能分析}

图 6(a) 比较了 GA、His-CDs/GA-1、His-CDs/ GA-2 和 His-CDs/GA-3 在扫速 $10 \mathrm{mV} \cdot \mathrm{s}^{-1}$, 电压窗口 为 $-1 \sim 0 \mathrm{~V}$ 下的 $\mathrm{CV}$ 曲线。由图可见, 所有电极的 $\mathrm{CV}$ 曲线呈现类矩形形状。值得注意的是，相对于 GA, His-CDs/GA-1、His-CDs/GA-2 和 His-CDs/GA-3 的 $\mathrm{CV}$ 曲线在 $-0.6 \sim-0.7 \mathrm{~V}$ 处出现了较弱的氧化还原峰, 
这主要由于 His-CDs 中丰富的含氮和含氧基团在碱 性电解质中发生了氧化还原反应 ${ }^{[5,23]}$ 。此外, His$\mathrm{CDs} / \mathrm{GA}-2$ 的 CV 曲线积分面积明显大于其它电极, 表明其具有更高的比电容。图 6(b)为所有电极在 $1 \mathrm{~A} \cdot \mathrm{g}^{-1}$ 下的充放电曲线, 曲线呈现出轻微变形的三 角形形状, 表明电极的比电容以双电层电容为主, 同时还存在赝电容, 这与 $\mathrm{CV}$ 结果相一致。通过公式 (S1)计算 GA、His-CDs/GA-1、His-CDs/GA-2 和 His$\mathrm{CDs} / \mathrm{GA}-3$ 的比电容分别为 $172 、 254 、 304 、 215 \mathrm{~F} \cdot \mathrm{g}^{-1}$ 。 相比较于 GA, His-CDs/GA-1、His-CDs/GA-2 和 His-CDs/GA-3 的比电容分别提高了 $47.7 \% 、 76.7 \%$ 、 $25.0 \%$ 。这主要归功于以下三点: (1)His-CDs 的引入 提高了复合材料的有效比表面积, 增加了电活性位 点; (2)His-CDs 边缘含有大量的咪唑基等含氮基团, 它们作为给电子基团, 能够在石墨烯表面形成富电 子区域, 有利于吸附更多的电解液阳离子, 从而改 善比电容 ${ }^{[19]}$; (3)His-CDs/GA 中的含氮和含氧基团 为复合材料提供了额外的噟电容。此外, 随着 His$\mathrm{CDs}$ 添加量的增加, His-CDs/GA 的比电容先升高后 降低。结合图 S4 样品的 BET 分析, 原因主要是过 量的 His-CDs 发生团聚, 导致 His-CDs/GA 的比表面 积降低, 进而使得电解液离子吸/脱附位点减少。
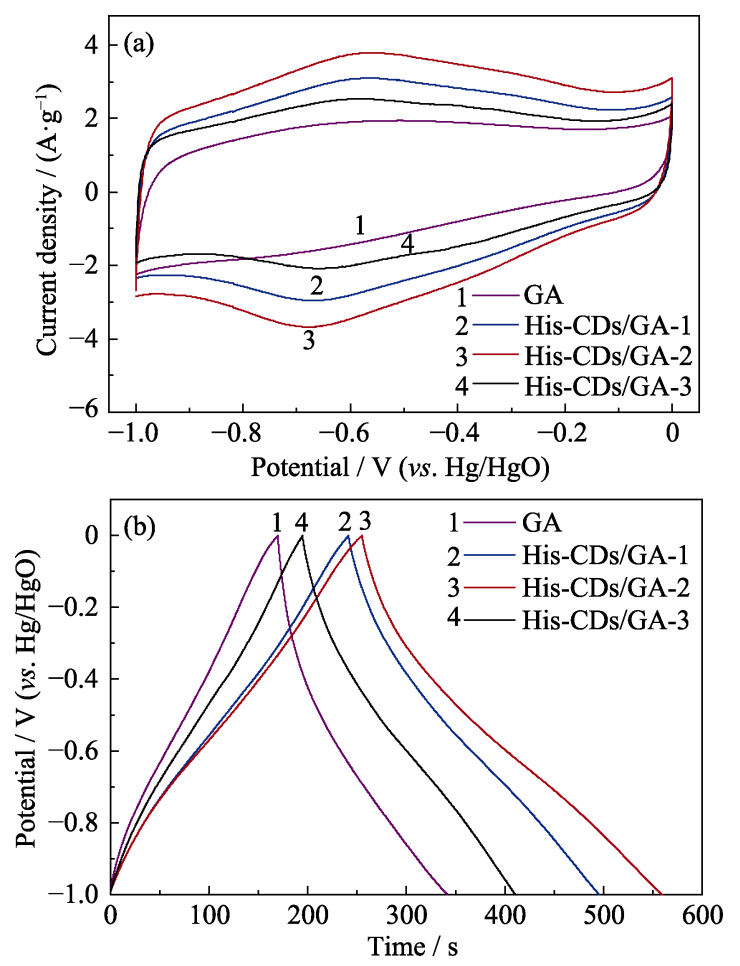

图 6 GA、His-CDs/GA-1、His-CDs/GA-2 和 His-CDs/GA-3 在 $10 \mathrm{mV} \cdot \mathrm{s}^{-1}$ 扫描速率下的循环伏安曲线(a)和 $1 \mathrm{~A} \cdot \mathrm{g}^{-1}$ 电流密 度下的恒流充放电曲线(b)

Fig. $6 \mathrm{CV}$ curves (a) at $10 \mathrm{mV} \cdot \mathrm{s}^{-1}$ and $\mathrm{GCD}$ curves (b) at $1 \mathrm{~A} \cdot \mathrm{g}^{-1}$ of GA, His-CDs/GA-1, His-CDs/GA-2, and His-CDs/ GA-3
His-CDs/GA-2 在不同扫描速率下的 CV 曲线列于 图 7(a), 当扫描速率从 $5 \mathrm{mV} \cdot \mathrm{s}^{-1}$ 增加到 $100 \mathrm{mV} \cdot \mathrm{s}^{-1}$ 时, CV 曲线形状并未发生明显变化, 表明 His-CDs/ GA-2 具有较低的电阻和良好的倍率性能。图 7(b) 为 His-CDs/GA-2 在不同电流密度下的 GCD 曲线。 所有的 GCD 曲线均未出现明显的电压降, 进一步 说明 His-CDs/GA-2 具有出色的电荷转输能力。由公 式(S1)计算出 His-CDs/GA-2 在电流密度 $1 、 2 、 3 、 5 、$ $10 、 20 、 30$ 和 $50 \mathrm{~A} \cdot \mathrm{g}^{-1}$ 下的比电容分别为 $304 、 282$ 、 $272 、 258 、 243 、 231 、 224$ 和 $217 \mathrm{~F} \cdot \mathrm{g}^{-1}$ 。三电极体 系下，与先前所报道的石墨烯基电极材料的比电容 相比较(如表 S1 所示), His-CDs/GA-2 展现出更高的 比电容, 表明其具备在高性能超级电容器应用的潜 能。图 7(c)为所有电极的倍率性能图。当电流密度 从 $1 \mathrm{~A} \cdot \mathrm{g}^{-1}$ 增加到 $50 \mathrm{~A} \cdot \mathrm{g}^{-1}$ 时, His-CDs/GA-1、His$\mathrm{CDs} / \mathrm{GA}-2$ 和 His-CDs/GA-3 的比电容保持率分别为 $65.4 \% 、 71.4 \%$ 和 $62.3 \%$, 均高于 $\mathrm{GA}(57.6 \%)$ 。 His$\mathrm{CDs} / \mathrm{GA}$ 倍率性能的提高, 主要归功于 His-CDs 增 加了石墨烯片的层间距以及其边缘的含氮和含氧基 团增强了活性材料的润湿性, 从而加快了电解液离 子的扩散 ${ }^{[8,11]}$ 。图 7(d) 为 GA 和 His-CDs/GA-2 的循 环性能图。GA 和 His-CDs/GA-2 在 $10 \mathrm{~A} \cdot \mathrm{g}^{-1}$ 下, 循 环充放电 30000 次后，比电容保持率分别为 $90.8 \%$ 和 $93.5 \%$ 。结果表明 His-CDs 负载在石墨烯片上, 提 高了电极的循环稳定性。值得注意的是, His-CDs/ GA-2 在前 6000 次循环, 其比电容损失率为 $4.9 \%$, 后 24000 次仅损失了 $1.6 \%$ 。这主要是由于在前 6000 次循环中, His-CDs/GA-2 表面不稳定活性基团的脱 落导致了赝电容的损失。

4 种电极的交流阻抗图谱如图 S6 所示。His$\mathrm{CDs} / \mathrm{GA}$ 的 $R_{\mathrm{s}}$ 和 $R_{\mathrm{ct}}$ 均小于 GA, 说明添加 His-CDs 能够降低复合材料的等效串联电阻和电荷转移阻 抗。采用四探针法测得 His-CDs/GA-1、His-CDs/GA-2 和 His-CDs/GA-3 的电导率分别为 $64.3 、 82.6$ 和 $49.6 \mathrm{~S} \cdot \mathrm{m}^{-1}$, 均高于 $\mathrm{GA}\left(35.8 \mathrm{~S} \cdot \mathrm{m}^{-1}\right)$ 。提高电导率有 助于充放电过程中电荷的快速传输, 改善电极的倍 率性能, 这与上述 $\mathrm{CV}$ 和 $\mathrm{GCD}$ 测试的结果相一致。

\section{4 两电极电化学性能分析}

为进一步研究 His-CDs/GA-2 复合电极材料的实 际应用, 将 His-CDs/GA-2 组装成对称超级电容器 (His-CDs/GA-2//His-CDs/GA-2), 在两电极体系下测 试。图 $S 7$ (a) 为该对称电容器在电压窗口为 $0 \sim 1 \mathrm{~V}$, 扫 描速率 5 100 $\mathrm{mV} \cdot \mathrm{s}^{-1}$ 下的 $\mathrm{CV}$ 曲线。由图可见, 在高 扫描速率 $100 \mathrm{mV} \cdot \mathrm{s}^{-1}$ 下, $\mathrm{CV}$ 曲线的形状仅出现轻微 的偏斜, 表明其具有良好的电荷传输能力。图 S7(b) 

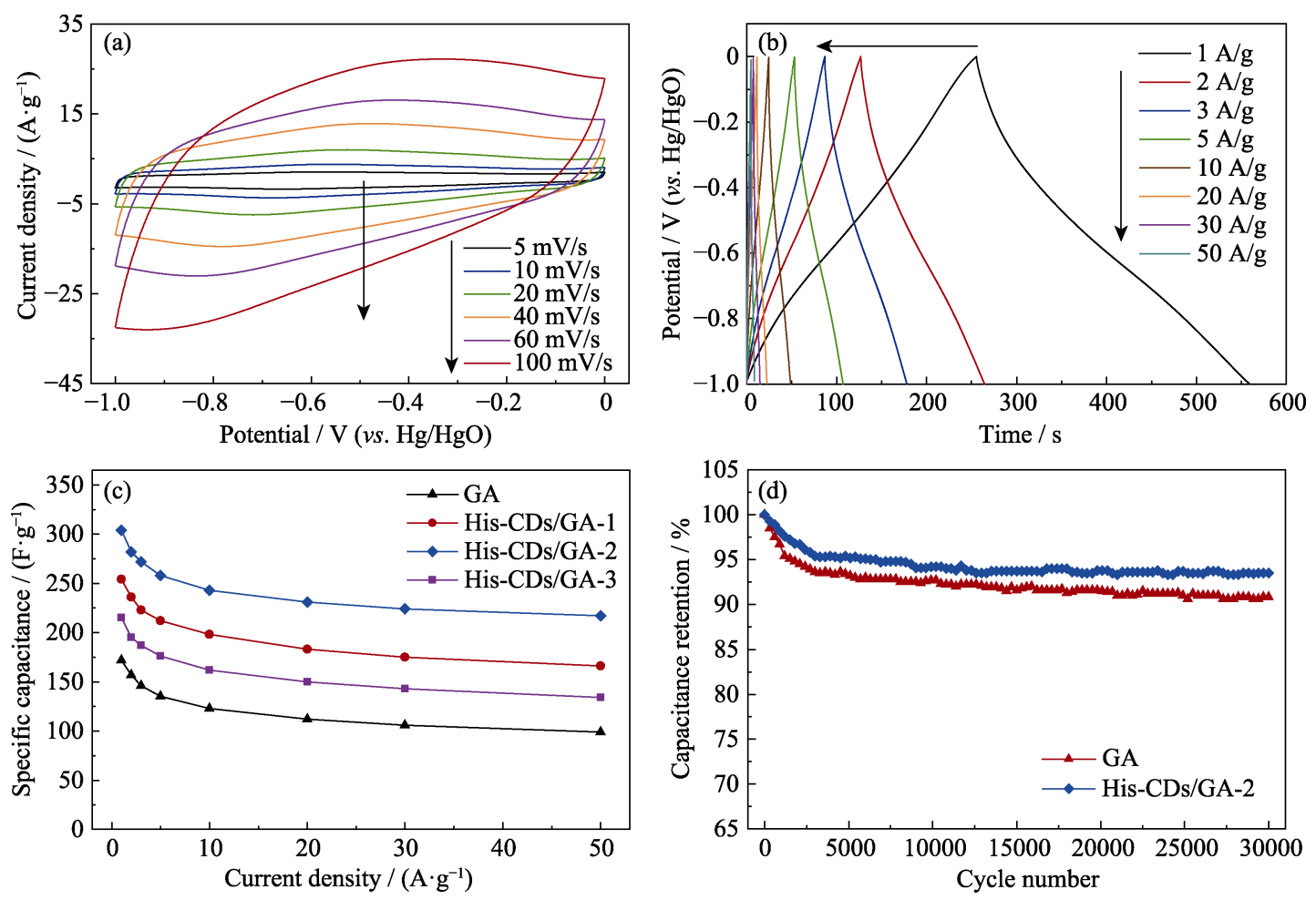

图 7 His-CDs/GA-2 在不同扫描速率下的循环伏安曲线(a)和在不同电流密度下的充放电曲线(b); GA、His-CDs/GA-1、

His-CDs/GA-2 和 His-CDs/GA-3 的倍率性能图(c); GA 和 His-CDs/GA-2 在 $10 \mathrm{~A} \cdot \mathrm{g}^{-1}$ 下的循环性能图(d)

Fig. 7 CV curves at different scan rates (a) and GCD curves at different current densities (b) of His-CDs/GA-2; Rate capabilities of GA, His-CDs/GA-1, His-CDs/GA-2 and His-CDs/GA-3 (c); Cycling performances of GA and His-CDs/GA-2 at 10 A-g ${ }^{-1}$ (d)

为 His-CDs/GA-2//His-CDs/GA-2 在不同电流密度下 的 $\mathrm{GCD}$ 曲线。由图可见，所有 $\mathrm{GCD}$ 曲线均呈现良 好的对称性, 且未出现明显的电压降。图 $\mathrm{S} 8$ 为 His-CDs/GA-2//His-CDs/GA-2 的循环性能图。该对 称电容器在 $5 \mathrm{~A} \cdot \mathrm{g}^{-1}$ 下, 循环充放电 20000 次, 比电 容保持率为 $88.4 \%$ 。His-CDs/GA-2//His-CDs/GA-2 良好的循环保持率主要得益于其独特的三维多孔结 构有效缓解了充放电过程中体积的膨胀和收缩。基 于 GCD 曲线, 由公式(S2 S4)计算对称电容器的能 量密度和功率密度, 结果如图 8 所示。该装置在功 率密度为 $250 \mathrm{~W} / \mathrm{kg}$ 时, 能量密度达到 $10.14 \mathrm{Wh} / \mathrm{kg}$,

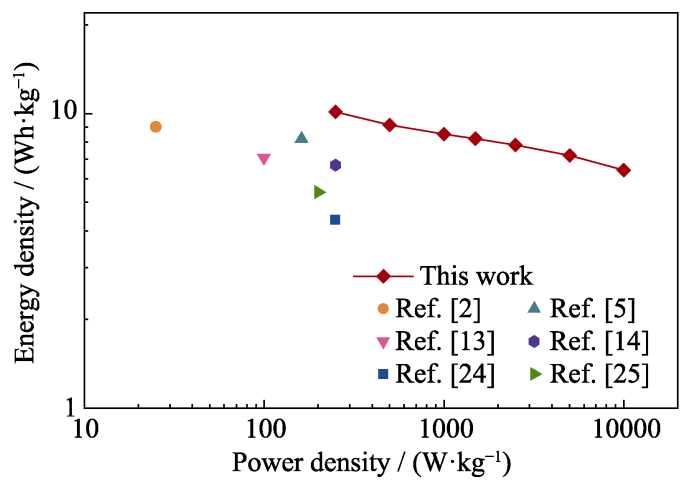

图 8 His-CDs/GA-2//His-CDs/GA-2 的拉贡图

Fig. 8 Ragon plots of His-CDs/GA-2//His-CDs/GA-2
且在功率密度增加到 $10 \mathrm{~kW} / \mathrm{kg}$ 时, 能量密度仍能保 持 $6.43 \mathrm{Wh} / \mathrm{kg}$ 。此外, His-CDs/GA-2//His-CDs/GA-2 最大能量密度高于先前文献所报道的石墨烯基对称 电容器 ${ }^{[2,5,13-14,24-25] 。 ~}$

\section{3 结论}

通过一步水热法制备出组氨酸功能化碳点/石 墨烯气凝胶。His-CDs 均匀负载在石墨烯片上, 有效 抑制了石墨烯片的团聚，从而增大复合材料的比表 面积。此外, His-CDs/GA-2 表面丰富的含氧和含氮 基团, 能够加快电解液离子的渗透和扩散并提供额 外的噟电容。当 GO 与 His-CDs 质量比为 $2: 1$ 时, His-CDs $/ \mathrm{GA}-2$ 在 $1 \mathrm{~A} \cdot \mathrm{g}^{-1}$ 下比电容达到 $304 \mathrm{~F} \cdot \mathrm{g}^{-1}$; 当 电流密度从 $1 \mathrm{~A} \cdot \mathrm{g}^{-1}$ 增加到 $50 \mathrm{~A} \cdot \mathrm{g}^{-1}$, 倍率性能达到 $71.4 \%$; 在 $10 \mathrm{~A} \cdot \mathrm{g}^{-1}$ 下, 循环充放电 30000 次后, 其 比电容保持率为 $93.5 \%$ 。此外，基于 His-CDs/GA 的 对称超级电容器展现出高能量密度, 在功率密度为 $250 \mathrm{~W} / \mathrm{kg}$ 时, 能量密度达到 $10.14 \mathrm{Wh} / \mathrm{kg}$, 并具有良 好的循环稳定性, 在 $5 \mathrm{~A} \cdot \mathrm{g}^{-1}$ 下循环 20000 次后, 比 电容仍保持 $88.4 \%$ 。本工作为基于碳点和石墨烯的 电极材料在高性能超级电容器等其它储能器件中的 应用提供了方法和指导。 


\section{补充材料}

\author{
本文相关补充材料可登陆 https://doi.org/10.15541/ \\ jim20190630 查看。
}

\section{参考文献:}

[1] LI Z, WEI J J, REN J, et al. Hierarchical construction of highperformance all-carbon flexible fiber supercapacitors with graphene hydrogel and nitrogen-doped graphene quantum dots. Carbon, 2019, 154: 410-419.

[2] LIU D P, LI Q W, LI S, et al. A confinement strategy to prepare $\mathrm{N}$-doped reduced graphene oxide foams with desired monolithic structures for supercapacitors. Nanoscale, 2019, 11(10): 4362-4368.

[3] HUO J H, XUE Y J, ZHANG X J, et al. Hierarchical porous reduced graphene oxide decorated with molybdenum disulfide for high-performance supercapacitors. Electrochimica Acta, 2018, 292: 639-645.

[4] LI B, HU N T, SU Y T, et al. Direct inkjet printing of aqueous inks to flexible all-solid-state graphene hybrid micro-supercapacitors. ACS Applied Materials \& Interfaces, 2019, 11(49): 46044-46053.

[5] XIA K S, HUANG Z Y, ZHENG L, et al. Facile and controllable synthesis of N/P co-doped graphene for high-performance supercapacitors. Journal of Power Sources, 2017, 365: 380-388.

[6] YU D Y, ZHANG Z Q, MENG Y N, et al. The synthesis of hierarchical $\mathrm{ZnCo}_{2} \mathrm{O}_{4} @ \mathrm{MnO}_{2}$ core-shell nanosheet arrays on $\mathrm{Ni}$ foam for high-performance all-solid-state asymmetric supercapacitors. Inorganic Chemistry Frontiers, 2018, 5(3): 597-604.

[7] LI F, LIU J, MA Y, et al. A monolithic integrated ultra-flexible allsolid-state supercapacitor based on a polyaniline conducting polymer. Journal of Materials Chemistry A, 2019, 7(25): 15378-15386.

[8] HOANG V C, NGUYEN L H, GOMES V G. High efficiency supercapacitor derived from biomass based carbon dots and reduced graphene oxide composite. Journal of Electroanalytical Chemistry, 2019, 832: 87-96.

[9] WU Z Y, FAN L, TAO Y R, et al. Pomelo peel derived hierarchical porous carbon as electrode materials for high-performance supercapacitor. Chinese Journal of Inorganic Chemistry, 2018, 34(7): 1249-1260.

[10] CHENG H H, YI F Y, CAO A M, et al. Supermolecule self-assembly promoted porous $\mathrm{N}, \mathrm{P}$ co-doped reduced graphene oxide for high energy density supercapacitors. ACS Applied Energy Materials, 2019, 2(6): 4084-4091.

[11] HUANG Y L, GAO A M, SONG X N, et al. Supramolecule-inspired fabrication of carbon nanoparticles in situ anchored graphene nanosheets material for high-performance supercapacitors. ACS Applied Materials \& Interfaces, 2016, 8(40): 26775-26782.

[12] LI Q, CHENG H Y, WU X J, et al. Enriched carbon dots/graphene microfibers towards high-performance micro-supercapacitors. Jou- rnal of Materials Chemistry A, 2018, 6(29): 14112-14119.

[13] SUN X X, CHENG P, WANG H J, et al. Activation of graphene aerogel with phosphoric acid for enhanced electrocapacitive performance. Carbon, 2015, 92: 1-10.

[14] ZHAO M Y, CUI X X, XU Y S, et al. An ordered mesoporous carbon nanosphere-encapsulated graphene network with optimized nitrogen doping for enhanced supercapacitor performance. Nanoscale, 2018, 10(32): 15379-15386.

[15] ZHU S J, MENG Q N, WANG L, et al. Highly photoluminescent carbon dots for multicolor patterning, sensors, and bioimaging. Angewandte Chemie-International Edition, 2013, 52(14): 39533957.

[16] TANG Y, RAO L S, LI Z T, et al. Rapid synthesis of highly photoluminescent nitrogen-doped carbon quantum dots via a microreactor with foamy copper for the detection of $\mathrm{Hg}^{2+}$ ions. Sensors and Actuators B-Chemical, 2018, 258: 637-647.

[17] QU D, LIU J A, MIAO X et al. Peering into water splitting mechanism of g- $\mathrm{C}_{3} \mathrm{~N}_{4}$-carbon dots metal-free photocatalyst. Applied $\mathrm{Ca}$ talysis B-Environmental, 2018, 227: 418-424.

[18] ALAS M O, GUNGOR A, GENC R, et al. Feeling the power: robust supercapacitors from nanostructured conductive polymers fostered with $\mathrm{Mn}^{2+}$ and carbon dots. Nanoscale, 2019, 11(27): 12804- 12816.

[19] WEI J S, DING C, ZHANG P, et al. Robust negative electrode materials derived from carbon dots and porous hydrogels for highperformance hybrid supercapacitors. Advanced Materials, 2019, 31(5): 1806197.

[20] WANG H Y, YANG Y Q, ZHOU X Y, et al. $\mathrm{NiCo}_{2} \mathrm{~S}_{4} /$ tryptophanfunctionalized graphene quantum dot nanohybrids for highperformance supercapacitors. New Journal of Chemistry, 2017, 41(3): $1110-1118$.

[21] ZHAO Y F, RAN W, HE J, et al. Oxygen-rich hierarchical porous carbon derived from artemia cyst shells with superior electrochemical performance. ACS Applied Materials \& Interfaces, 2015, 7(2): 1132-1139.

[22] XU Y J, LI X Y, HU G H, et al. Graphene oxide quantum dot-derived nitrogen-enriched hybrid graphene nanosheets by simple photochemical doping for high-performance supercapacitors. Applied Surface Science, 2017, 422: 847-855.

[23] ZHAO X, DONG H W, XIAO Y, et al. Three-dimensional nitrogendoped graphene as binder-free electrode materials for supercapacitors with high volumetric capacitance and the synergistic effect between nitrogen configuration and supercapacitive performance. Electrochimica Acta, 2016, 218: 32-40.

[24] WANG M, YANG J, JIA K L, et al. Boosting supercapacitor performance of graphene by coupling with nitrogen-doped hollow carbon frameworks. Chemistry-A European Journal, 2020, 26(13): 2897-2903.

[25] HU Z X, LI S S, CHENG P P, et al. N, P-co-doped carbon nanowires prepared from bacterial cellulose for supercapacitor. Journal of Materials Science, 2016, 51(5): 2627-2633. 


\title{
补充材料:
}

\section{组氨酸功能化碳点/石墨烯气凝胶的制备及超级电容器性能}

\author{
丁卓峰 ${ }^{1}$, 杨永强 ${ }^{2}$, 李在均 ${ }^{1}$
}

(1. 江南大学 化学与材料工程学院, 无锡 214122; 2. 江苏省特种设备安全监督检验研究院无锡分院, 无锡 214071)

\section{S1 电极的制备及电化学性能测试}

将制备好的 GA 和 His-CDs/GA 在红外压片机 上用 $5 \mathrm{MPa}$ 的压力压成薄片, 并裁剪为 $1 \mathrm{~cm} \times 1 \mathrm{~cm}$ 的正方形。然后与清洗后的泡沫镍贴合, 在 $10 \mathrm{MPa}$ 压力下压实。每个电极的活性物质负载量为 2 $2.5 \mathrm{mg} \cdot \mathrm{cm}^{-2}$ 。三电极体系中, 以该电极片作为工作 电极, 铂片 $(1 \mathrm{~cm} \times 1 \mathrm{~cm})$ 作为对电极, $\mathrm{Hg} / \mathrm{HgO}$ 电极作 为参比电极, $6 \mathrm{~mol} \cdot \mathrm{L}^{-1} \mathrm{KOH}$ 溶液作为电解液, 在电 化学工作站(上海辰华, CHI 660D 型)上进行循环伏 安 $(\mathrm{CV})$ 和恒电流充放电 $(\mathrm{GCD})$ 测试, 电压窗口为 $-1 \sim 0 \mathrm{~V}$ 。质量比电容 $C_{\mathrm{s}}\left(\mathrm{F} \cdot \mathrm{g}^{-1}\right)$ 由以下公式计算:

$$
C_{\mathrm{s}}=\frac{I \times \Delta t}{m \times \Delta V}
$$

其中, $I(\mathrm{~A})$ 为放电电流, $\Delta t(\mathrm{~s})$ 为放电时间, $m(\mathrm{~g})$ 为活 性材料质量, $\Delta V(\mathrm{~V})$ 为减去电压降后的电压窗口值

两电极体系中, 将两片质量相同的 His-CDs/ GA-2 电极作为一组工作电极, $6 \mathrm{~mol} \cdot \mathrm{L}^{-1} \mathrm{KOH}$ 溶液 作为电解液, 纤维素膜 (NKK, MPF30AC) 作为隔膜, 依次装入到纽扣式电池壳(CR 2032 型)中, 组装成 纽扣式对称电容器。在电化学工作站上进行 $\mathrm{CV}$ 和 $\mathrm{GCD}$ 测试, 电压窗口为 $0 \sim 1 \mathrm{~V}$ 。在武汉蓝电电池测 试系统(LAND CT2001A)上进行循环性能测试。对 称电容器的总质量比电容 $C\left(\mathrm{~F} \cdot \mathrm{g}^{-1}\right)$ 由以下公式计算:

$$
C=\frac{I \times \Delta t}{m \times \Delta V}
$$

能量密度 $E(\mathrm{Wh} / \mathrm{kg})$ 和功率密度 $P(\mathrm{~W} / \mathrm{kg})$ 由以下

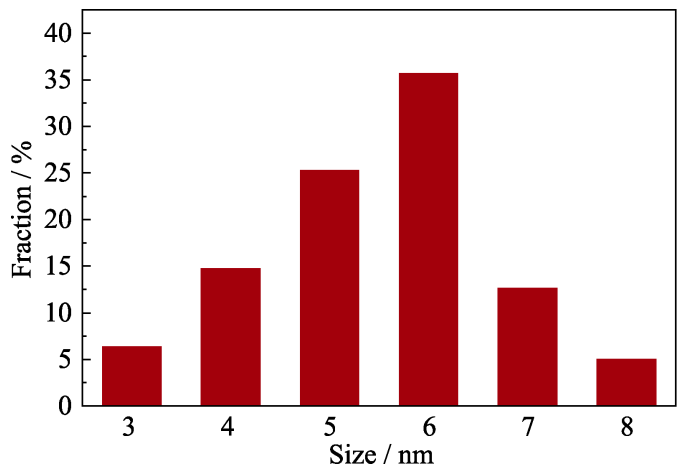

图 S1 His-CDs 的尺寸分布图

Fig. S1 Size distribution of His-CDs
公式计算:

$$
\begin{gathered}
E=\frac{0.5 C(\Delta V)^{2}}{3.6} \\
P=\frac{E \times 3600}{\Delta t}
\end{gathered}
$$
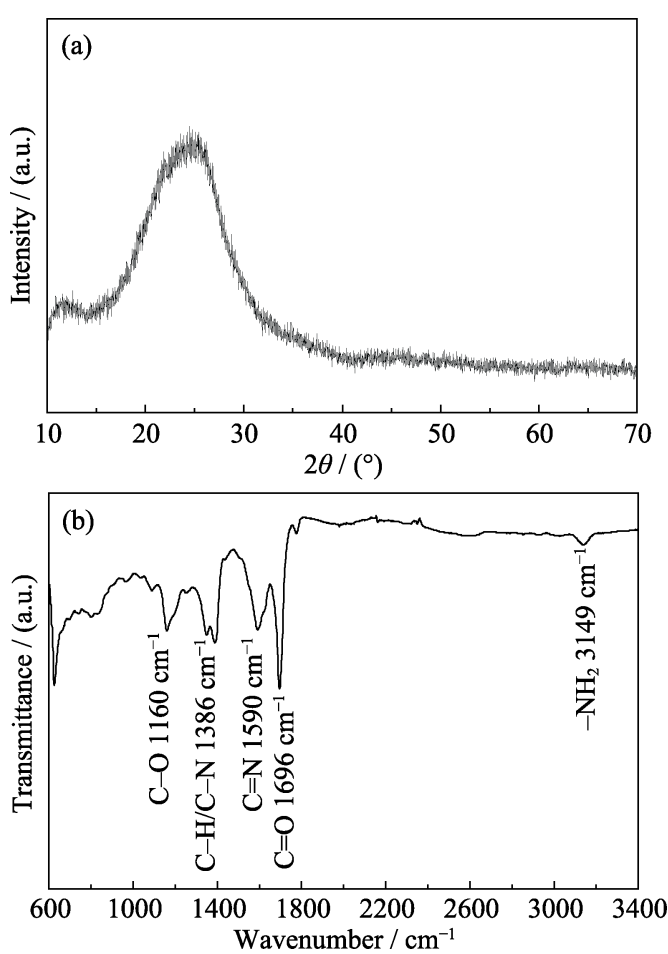

图 S2 His-CDs 的 XRD 图谱(a)和红外光谱图(b)

Fig. S2 XRD pattern (a) and IR spectrum (b) of His-CDs

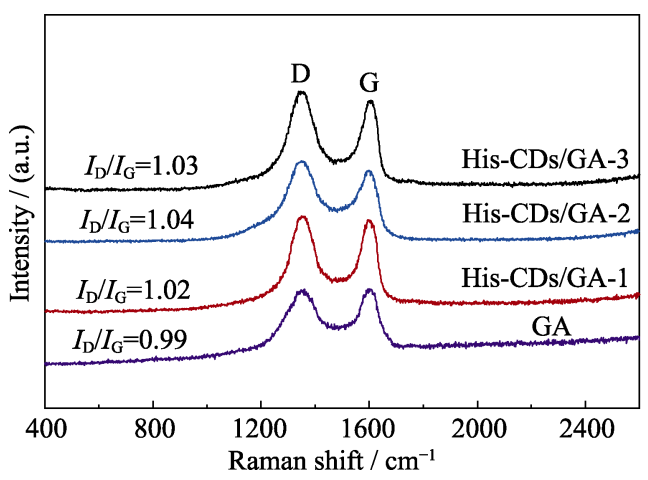

GA、His-CDs/GA-1、His-CDs/GA-2 和 His-CDs/GA-3 的拉曼谱图

Fig. S3 Raman spectra of GA, His-CDs/GA-1, His-CDs/GA-2 and His-CDs/GA-3 

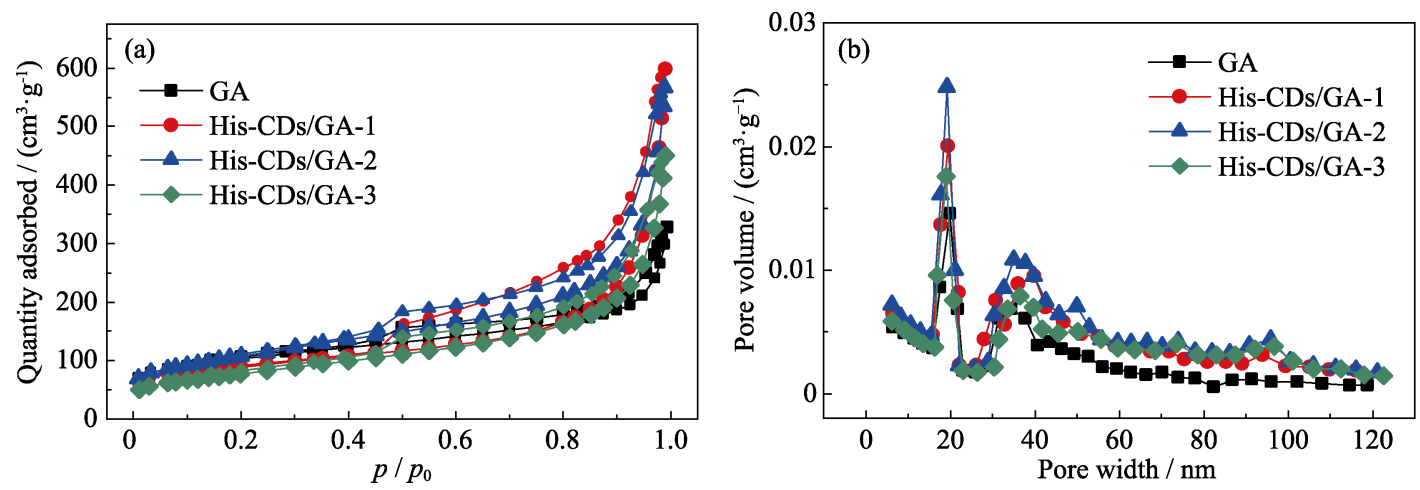

图 S4 GA、His-CDs/GA-1、His-CDs/GA-2 和 His-CDs/GA-3 的氮气吸附/脱附等温线(a)和孔径分布曲线(b)

Fig. S4 $\mathrm{N}_{2}$ absorption-desorption isotherms (a) and pore size distributions (b) of GA, His-CDs/GA-1, His-CDs/GA-2 and His-CDs/GA-3
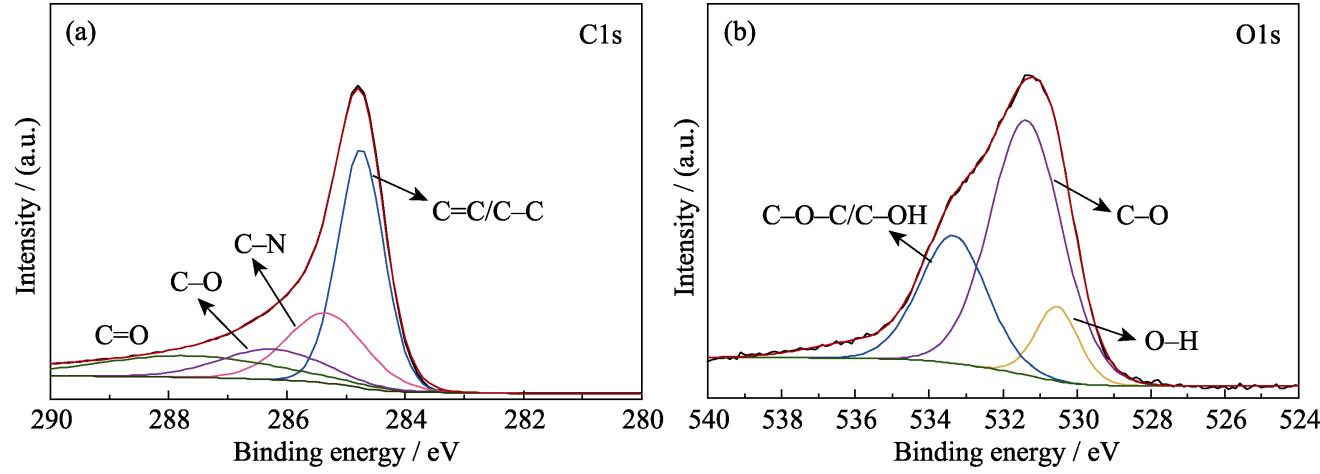

图 S5 His-CDs/GA-2 的 C1s(a)和 O1s(b) XPS 图谱

Fig. S5 C1s (a) and O1s (b) XPS spectra of His-CDs/GA-2

表 S1 His-CDs/GA-2 与其它石墨烯基电极比电容的比较

Table S1 Comparison of the specific capacitance of His-CDs/GA-2 and other graphene-based electrodes

\begin{tabular}{|c|c|c|c|}
\hline Electrode & $\begin{array}{c}\text { Specific } \\
\text { capacitance/ } \\
\left(\mathrm{F} \cdot \mathrm{g}^{-1}\right)\end{array}$ & $\begin{array}{l}\text { Current } \\
\text { density/ } \\
\left(\mathrm{A} \cdot \mathrm{g}^{-1}\right)\end{array}$ & Ref. \\
\hline $\mathrm{RGO} / \mathrm{CD}$ & 278 & 0.2 & [1] \\
\hline N-OMCN@GN & 242.3 & 1 & [2] \\
\hline N/S-rGAs & 180.5 & 1 & [3] \\
\hline GQDs-3DG & 268 & 1.25 & [4] \\
\hline GQD/3DG & 242 & 1.17 & [5] \\
\hline 3D graphene layers & 231.2 & 1 & [6] \\
\hline $\begin{array}{c}\text { 3D bubble-like } \\
\text { graphene frameworks }\end{array}$ & 277 & 1 & [7] \\
\hline His-CDs/GA-2 & 304 & 1 & This work \\
\hline
\end{tabular}

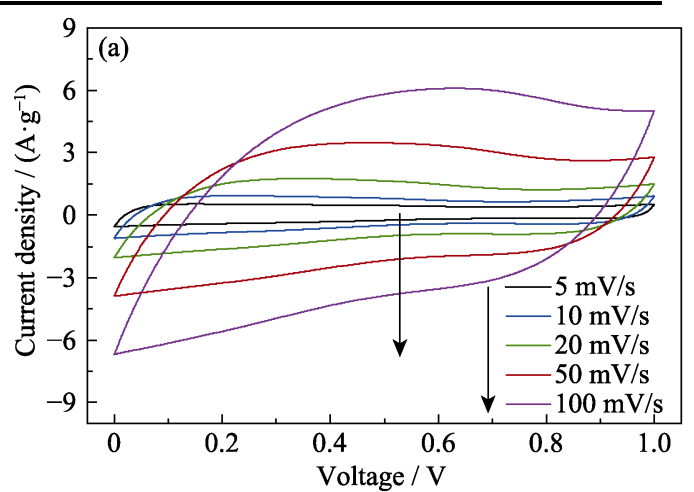

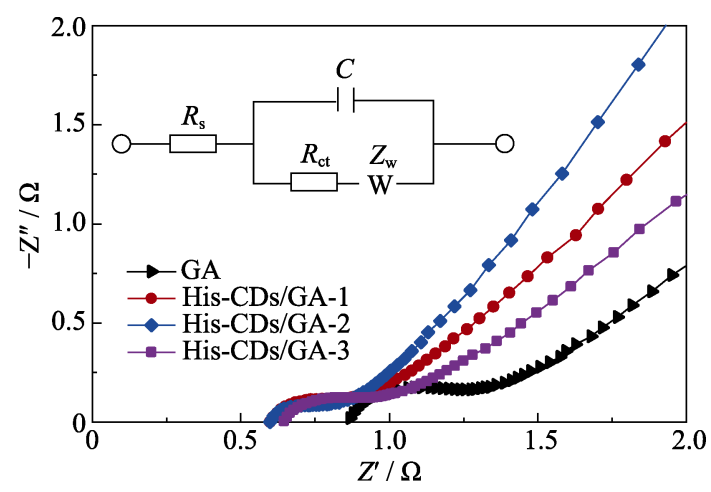

图 S6 4 种电极的的交流阻抗图谱, 插图为 EIS 测量的等效 电路

Fig. S6 AC impedance plots of 4 electrodes with inset showing the equivalent ciruit from the EIS measurements

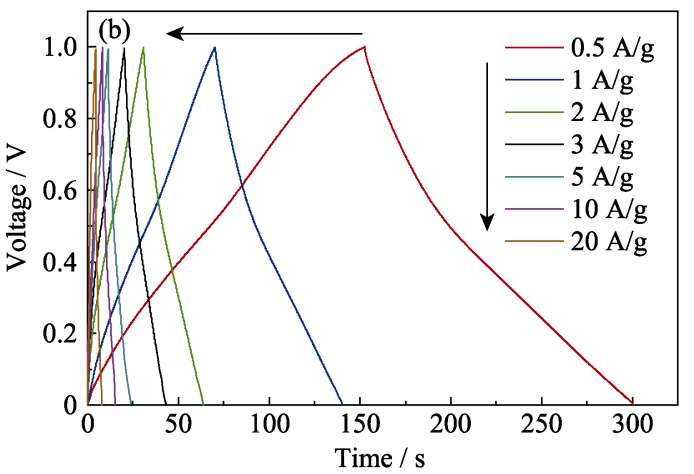

图 S7 His-CDs/GA-2//His-CDs/GA-2 对称电容器在不同扫描速率下的循环伏安曲线(a)和在不同电流密度下的充放电曲线(b)

Fig. S7 CV curves (a) at different scan rates and GCD curves (b) at different current densities of His-CDs/GA-2//His-CDs/ GA-2 symmetrical supercapacitor 


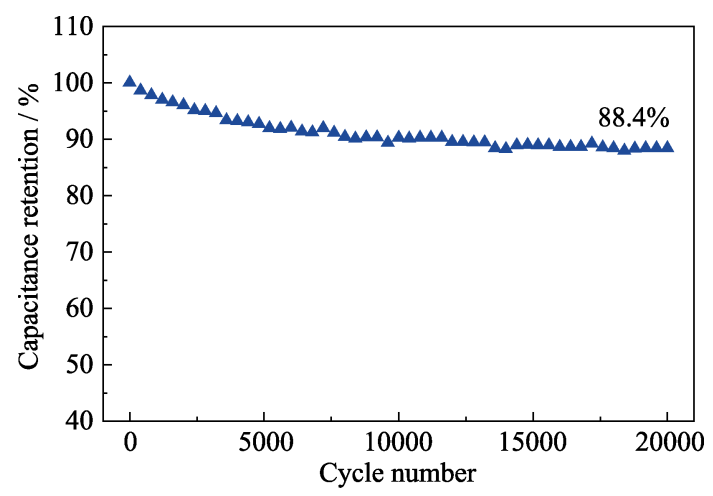

图 S8 His-CDs/GA-2//His-CDs/GA-2 对称电容器在 $5 \mathrm{~A} \cdot \mathrm{g}^{-1}$ 下循环 20000 次的循环性能图

Fig. S8 Cycling performance at $5 \mathrm{~A} \cdot \mathrm{g}^{-1}$ over 20000 cycles of His-CDs/GA-2//His-CDs/GA-2 symmetrical supercapacitor

\section{参考文献:}

[1] HOANG V C, NGUYEN L H, GOMES V G. High efficiency supercapacitor derived from biomass based carbon dots and reduced graphene oxide composite. Journal of Electroanalytical Chemistry,
2019, 832: 87-96.

[2] CHENG H H, YI F Y, CAO A M, et al. Supermolecule self-assembly promoted porous N, P co-doped reduced graphene oxide for high energy density supercapacitors. ACS Applied Energy Materials, 2019, 2(6): 4084-4091.

[3] CHEN Y J, SUN L, LIU I E, et al. Synthesis of nitrogen/sulfur co-doped reduced graphene oxide aerogels for high-performance supercapacitors with ionic liquid electrolyte. Materials Chemistry and Physics, 2019, 238: 121932.

[4] CHEN Q, HU Y, HU C G, et al. Graphene quantum dots-threedimensional graphene composites for high-performance supercapacitors. Physical Chemistry Chemical Physics, 2014, 6(36): 19307-19313.

[5] LUO P H, GUAN X F, YU Y L, et al. Hydrothermal synthesis of graphene quantum dots supported on three-dimensional graphene for supercapacitors. Nanomaterials, 2019, 9(2): 1-10.

[6] HAO J N, LIAO Y Q, ZHONG Y Y, et al. Three-dimensional graphene layers prepared by a gas-foaming method for supercapacitor applications. Carbon, 2015, 94: 879-887.

[7] DUAN H Y, YAN T T, LI Z Y, et al. Rapid synthesis of self-supported three dimensional bubble-like graphene frameworks as highperformance electrodes for supercapacitors. Sustainable Energy \& Fuels, 2017, 1(7): 1557-1567. 